\title{
Model Merging Method Based on Fast Topology Coloring and Device Fuzzy Matching
}

\author{
Feng Li, Lingling Pan, Yijun Yu, Shuhai Feng \\ China Electric Power Research Institute \\ Nanjing, China
}

\begin{abstract}
Model merging method is used to form a large power grid simulation model and to test dispatching system functions. A model merging method based on power grid model file that meets CIM-E standard is proposed, and fast topology coloring separates inner-grid from the whole file-based power grid model. According to edit distance, device name fuzzy matching method for different grid models is given, and then grid model merging is achieved. The simulation results demonstrate that the proposed method is available.
\end{abstract}

Keywords—topology coloring; model merging; edit distance; fuzzy matching

\section{INTRODUCTION}

The test platform [1-2] provides parallel test services for multi-dispatching, multi-version dispatching system, and supports to test one or more dispatching systems at the same time. Test platform provides initial power grid model and operation mode for the dispatching system to be tested, which is a black box for the test platform. The test platform simulates real-time operation of power grid in test process, and sets disturbances by cases. It sends power grid data and the secondary signal after the disturbance to dispatching system by simulated front in real time. The dispatching system should do data processing based on these input data, and returns various types of process data to test platform to validate the results.

It needs to provide a realistic simulation environment for dispatching system to be test. The simulation model of power grid is primary problem to be solved. The test platform provides parallel test for multi-dispatching system such as state, regional, provincial and local dispatching systems. Therefore, the simulation model must cover the whole power grid models. The device information such as key ID needs to retain to interact with the simulation data and dispatching data. The upper dispatching department gathers the power grid model file from all subordinate dispatching departments, and merges these models. Reference [3] discusses model merging method from standard CIM61970, and proposes an online distributed modeling system design scheme of china southern power grid. Reference [4] studies the space-time coordinated modeling system from the standardized management, and proposes an online model merging method for D5000 system. Reference [5] updates the model connection incremental via updating the model incremental based on online model merging. However, these methods are inflexible to build model for dispatching system function test.
Common power grid model (CIM-E) is mainly used as model exchange format [6], and the document includes power grid model, parameters and operation mode. The grid model files are exported from each dispatching system, which are used for model merging. It provides better convenience to build power grid simulation model. The dispatching system model is usually a part of the large simulation grid model. It needs to associate it with the large grid devices, which can obtain all needed device data from the large grid simulation data set for dispatching system test. Therefore, the matching relationship between different grid models needs to be considered while merging grid models.

For the reasons above, the multi-dispatching model merging method based on CIM-E file is proposed in this paper. Firstly, the fast topology coloring [7-8] is used to separate inner-grid from the whole grid model, which is a critical technology. And then the multi-dispatching model file merging method is given. Finally, a device fuzzy matching method is put forward to achieve the device connection of EMS system and large grid model.

\section{FAST TOPOLOGY COLORING}

\section{A. Basic conception of power grid}

Power grid consists of nodes and lines from the view of geometry topology. The nodes are connection points of devices, and lines are two-terminal or multi-terminal devices, such as AC lines, transformers, switches, breakers, series capacitive and reactance, etc. The single-terminal devices cannot be considered, such as generators, loads, shunt capacitive and reactance, etc. They just hang to the connecting point of lines or buses and don't affect the topological analysis. The topology analysis aims at AC system, and HVDC system can be ignored.

The whole power grid can be abstracted as geometry topologies with points and lines, which can be described as $\mathrm{G}(\mathrm{V}, \mathrm{E})$ (V: Vertex point, E: Edge connection line). Independent connected graphs are identified and separated according to connection lines.

Geometry graph $G(V, E)$ includes node set and line set. Node set is the physical node information described by CIM-E file, and line set contains two-terminal or multi-terminal devices without tie lines given by CIM-E file. The E records are generated by both ends node (Vs, Ve) of lines, two-volume transformers, series capacitive and reactance. A three-volume 
transformer can be view as three two-volume transformers, and each two-volume transformer is an E record.

The topological analysis can be performed by $\mathrm{V}$ set and $\mathrm{E}$ set.

\section{B. Power grid partition process by topology colorin}

The power grid is divided by bottom-up topology coloring.

Topology color array int color [V.len] is defined as the same number of records V. Each element of the array corresponds to increment integer variable that assigned to each record $\mathrm{V}$, which is its initial topology color.

All records of connection line $\mathrm{E}$ are traversed. Assume Enow is the processed record of E set, and nodes Enow.Vs and Enow.Ve at both ends of Enow can be obtained. The topological color of these two nodes should be the same because they are connected with each other through the branch. Define the topological color of Enow.Vs is scolor $=$ color [Enow.Vs], and the topological color of Enow.Ve is ecolor = color [Enow.Ve]. Because Vs connects with Ve by Enow, the topological color $\mathrm{Ve}$ is same as $\mathrm{Vs}$, that color [Enow.Ve] = color [Enow.Vs]. All the nodes of connection lines are topologically colored until each connection line traversal is done.

Subnets can be achieved at the end of traversal. Based on the tie line branch E, all grid nodes are topological colored. Nodes with the same color belong to the same subnet, and it forms many connected graphs.

\section{Model Merging}

Many grid models need to be merged, and the original models are defined as subnet 1 , subnet 2, subnet n, etc. Each subnet is a part of the large interconnected power grid, and subnets connected together via connection devices. Lines, transformers, equivalent generator of lines, and equivalent load of transformers are connection devices. The subnet inner-grid model can be found out by fast topology coloring from boundary connection device, and then all other subnet innergrids are connected through connection devices to form a complete power grid model. The model merging flow is described as Figure 1.

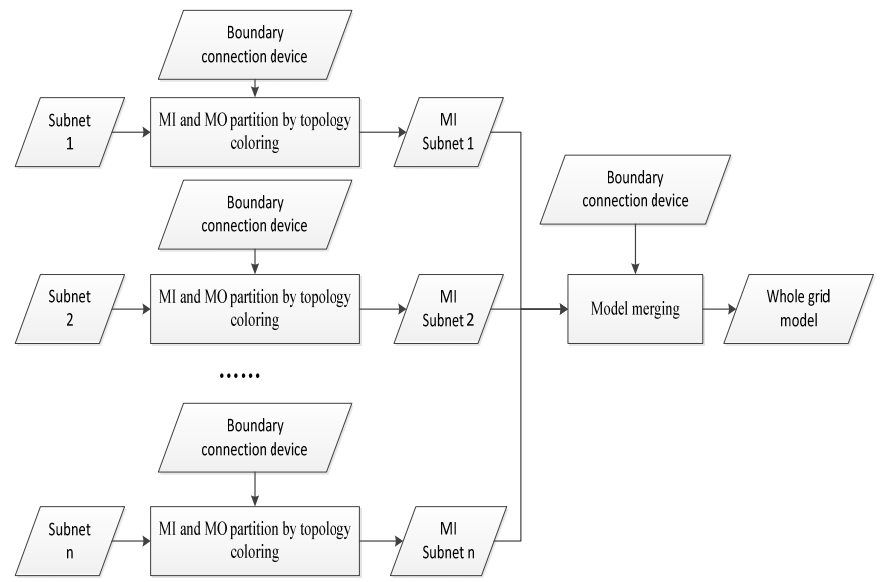

Fig.1 Flow chart of model merging based on topology coloring

\section{DeVice FuZZY MATChING}

The large merged grid model is used on test platform of the dispatching automation system as simulation model, and the calculated data are sent to EMS system model that contains subnet 1 . telemetry data and remote signalling data will be associated based on device's key ID. The subnet 1 includes two parts: inner-grid MI1 and outer-grid MO1. MI1 information is consistent with the large merged grid model, and MO1 is replaced by inner-grid MI2 of subnet 2, which requires model matching between MO1 and MI2. It is difficult to matching devices according to conventional methods, because there is no uniform device naming standard for different EMS system. In general, device matching can be achieved when device name is identical. We can analyze naming rules of EMS system and find a certain general rule, and then write codes to match device when parts of device name are the same. This method is cumbersome and error-prone. The fuzzy matching proposed in this paper can achieve high efficiency to match device name.

Edit distance, Levenshtein Distance, is usually used to describe the similarity degree of two strings. Edit distance $\mathrm{LD}(\mathrm{s} 1, \mathrm{~s} 2)$ represents the minimum operation times for single character such as insert, delete and replace in order to turn s1 into $\mathrm{s} 2$.

Two device names are generally matching if the string distance LSD between the two devices is the smallest distance comparing to LSD of other device name. However, it needs generous calculation large computation to achieve model matching between $\mathrm{MO} 1$ and MI2.

Taking into account the physical attributes of power system, station name and voltage classes are used to screen matching device, and large matrices can be converted a large number of small-dimension matrix to match device name. For example, line has the same station name at both ends and voltage level with the matching one, and breaks have the same station name and voltage level with the matching ones. Few devices in different sub-networks have different station name and voltage level, distances are edited by string for matching devices, and the matching results are checked manually. Device matching is according to device types

\section{NUMERICAL EXAMPLE}

The topology diagram of IEEE 9-bus system is shown in Figure 2. $E\{8,9\}$ and $E\{4,5\}$ are defined as open-end boundary tie lines.

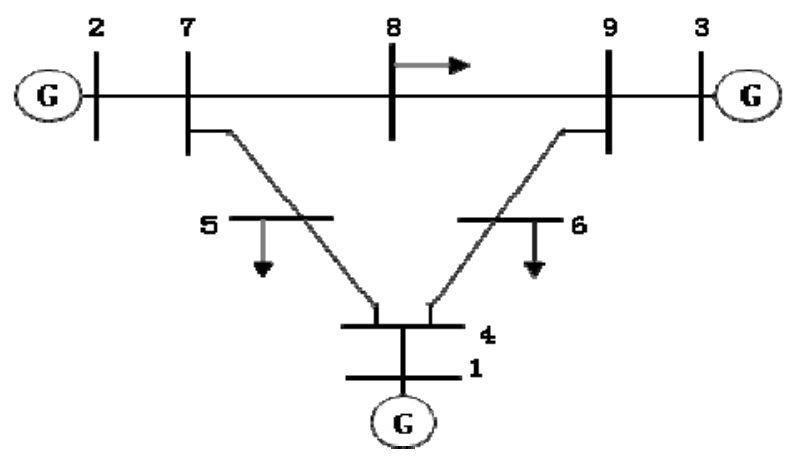

Fig. 2 Topology Diagram of IEEE 9-bus System 
$\mathrm{E}\{1,4\}, \mathrm{E}\{2,7\}, \mathrm{E}\{7,8\}, \mathrm{E}\{7,5\}, \mathrm{E}\{4,5\}, \mathrm{E}\{4,6\}, \mathrm{E}\{6,9\}$, $\mathrm{E}\{9,3\}, \mathrm{E}\{8,9\}$ are tie lines.

c\#num $\{n 1, n 2, n 3 \ldots\}$ describes node set $\{n 1, n 2, n 3, \ldots\}$ of topology color num, and c\#num $\{\varphi\}$ shows empty node set of topology color num.

These node have their own topology colors in original state, which are $\mathrm{c} 1\{1\}, \mathrm{c} 2\{2\}, \mathrm{c} 3\{3\}, \mathrm{c} 4\{4\}, \mathrm{c} 5\{5\}, \mathrm{c} 6\{6\}, \mathrm{c} 7\{7\}$, $\mathrm{c} 8\{8\}, \mathrm{c} 9\{9\}$.

Except open-end boundary tie lines $\mathrm{E}\{4,5\}, \mathrm{E}\{8,9\}$, tie lines $\mathrm{E}\{1,4\}, \mathrm{E}\{2,7\}, \mathrm{E}\{7,8\}, \mathrm{E}\{7,5\}, \mathrm{E}\{4,6\}, \mathrm{E}\{6,9\}, \mathrm{E}\{9,3\}$ are scanned successively.

Update the topology color from $\mathrm{E}\{1,4\}$, and the coloring information is described as,

$\mathrm{c} 1\{1,4\}, \mathrm{c} 2\{2\}, \mathrm{c} 3\{3\}, \mathrm{c} 4\{\phi\}, \mathrm{c} 5\{5\}, \mathrm{c} 6\{6\}$, $\mathrm{c} 7\{7\}, \mathrm{c} 8\{8\}, \mathrm{c} 9\{9\}$.

Update the topology color from $\mathrm{E}\{2,7\}$, and the coloring information is described as,

$\mathrm{c} 1\{1,4\}, \mathrm{c} 2\{2,7\}, \mathrm{c} 3\{3\}, \mathrm{c} 4\{\phi\}, \mathrm{c} 5\{5\}, \mathrm{c} 6\{6\}$, c7 $\{\phi\}, \quad c 8\{8\}, \quad c 9\{9\}$.

Update the topology color from $\mathrm{E}\{7,8\}$, and the coloring information is described as,

$\mathrm{c} 1\{1,4\}, \mathrm{c} 2\{2,7,8\}, \mathrm{c} 3\{3\}, \mathrm{c} 4\{\phi\}, \mathrm{c} 5\{5\}, \mathrm{c} 6\{6\}$, c7 $\{\phi\}, \quad c 8\{\phi\}, \quad c 9\{9\}$.

Update the topology color from $\mathrm{E}\{7,5\}$, and the coloring information is described as,

$\mathrm{c} 1\{1,4\}, \mathrm{c} 2\{2,7,8,5\}, \mathrm{c} 3\{3\}, \mathrm{c} 4\{\phi\}, \mathrm{c} 5\{\phi\}$, $\mathrm{c} 6\{6\}, \mathrm{c} 7\{\phi\}, \mathrm{c} 8\{\phi\}, \mathrm{c} 9\{9\}$.

Update the topology color from $\mathrm{E}\{4,6\}$, and the coloring information is described as,

$\mathrm{c} 1\{1,4,6\}, \mathrm{c} 2\{2,7,8,5\}, \mathrm{c} 3\{3\}, \mathrm{c} 4\{\phi\}, \mathrm{c} 5\{\phi\}$, $\mathrm{c} 6\{\phi\}, \mathrm{c} 7\{\phi\}, \mathrm{c} 8\{\phi\}, \mathrm{c} 9\{9\}$.

Update the topology color from $\mathrm{E}\{6,9\}\}$, and the coloring information is described as,

$\mathrm{c} 1\{1,4,6,9\}, \mathrm{c} 2\{2,7,8,5\}, \mathrm{c} 3\{3\}, \mathrm{c} 4\{\phi\}, \mathrm{c} 5\{\phi\}$, c6 $\{\phi\}, \operatorname{cc} 7 \phi \phi, c 8\{\phi\}, c 9\{\phi\}$.

Update the topology color from $\mathrm{E}\{9,3\}$, and the coloring information is described as,

$\mathrm{c} 1\{1,4,6,9,3\}, \mathrm{c} 2\{2,7,8,5\}, \mathrm{c} 3\{\phi\}, \mathrm{c} 4\{\phi\}, \mathrm{c} 5\{\phi\}$, c6 $\{\phi\}, \mathrm{c} 7\{\phi\}, \mathrm{c} 8\{\phi\}, \mathrm{c} 9\{\phi\}$.

The topology coloring is shown in Table 1. IEEE 9-bus system is divided into two subnets $\mathrm{c} 1\{1,4,6,9,3\}$ and $\mathrm{c} 2\{2,7,8,5\}$ by topology coloring with open-end tie lines $\mathrm{E}\{8,9\}$ and $\mathrm{E}\{4,5\}$.
Table 1 Node Coloring of Topology Analysis

\begin{tabular}{|c|c|c|c|c|c|c|c|c|c|}
\hline $\mathrm{E}$ & $\mathrm{V} 1$ & $\mathrm{~V} 2$ & $\mathrm{~V} 3$ & $\mathrm{~V} 4$ & $\mathrm{~V} 5$ & $\mathrm{~V} 6$ & $\mathrm{~V} 7$ & $\mathrm{~V} 8$ & $\mathrm{~V} 9$ \\
\hline 0 & 1 & 2 & 3 & 4 & 5 & 6 & 7 & 8 & 9 \\
\hline 1 & 1 & 2 & 3 & 1 & 5 & 6 & 7 & 8 & 9 \\
\hline 2 & 1 & 2 & 3 & 1 & 5 & 6 & 2 & 8 & 9 \\
\hline 3 & 1 & 2 & 3 & 1 & 5 & 6 & 2 & 2 & 9 \\
\hline 4 & 1 & 2 & 3 & 1 & 2 & 6 & 2 & 2 & 9 \\
\hline 5 & 1 & 2 & 3 & 1 & 2 & 1 & 2 & 2 & 9 \\
\hline 6 & 1 & 2 & 3 & 1 & 2 & 1 & 2 & 2 & 1 \\
\hline 7 & 1 & 2 & 1 & 1 & 2 & 1 & 2 & 2 & 1 \\
\hline
\end{tabular}

On this basis, the proposed method is used on a regional power grid. After merging regional and provincial power grid model, a large grid model that contains detailed provincial power grid model is formed as simulation model. The simulation data is provided to provincial dispatching system for test dispatching system functions, and the results confirm the validity of this method.

\section{CONCLUSION}

Many grid models need to be merged, and the original models are defined as subnet 1 , subnet 2, subnet n, etc. Each subnet is a part of the large interconnected power grid, and subnets connected together via connection devices. Lines, transformers, equivalent generator of lines, and equivalent load of transformers are connection devices. The subnet inner-grid model can be found out by fast topology coloring from boundary connection device, and then all other subnet innergrids are connected through connection devices to form a complete power grid model. The model merging flow is described as Figure 1.

Large grid simulation model that covers many areas is built for dispatching system test. The whole model merging method based on CIM-E standard files is proposed to adapt to the flexibility of simulation model establishment for test platform. The inner-grid model is obtained by topology coloring at grid simulation mod, and model merging is achieved. To solve the device name matching problem between large grid simulation model and test dispatching system model, the device fuzzy matching method based on the Levenshtein distance is put forward. These methods not only can be applied to test platform, but also can be applied to fast model building for power grid analysis.

\section{ACKNOWLEDGMENT}

This work was financially supported by SGCC Science and Technology Program "key technologies and application of test platform for dispatching automation system”.

\section{REFERENCES}

[1] F. Li, W.J. Zhuang, Y. Wang, et al. PSS/E-Based Simulation System Design for Functional Verification on the Power Dispatching Automation System. China Electric Power, 2014, 47(1):66-71.

[2] State Grid Company of China, Smart dispatching support system standard(D5000), 2010. 
[3] H.B. Sun, P. Li, M. Li, et al. Study and Design of Online Distributed Modeling System for China Southern Power Grid. Automation of Electric Power Systems, 2007, 31(10):82-86.

[4] W.M. Mi, L.X. Li, X.W. Shang, et al. An Investigation on Hierarchical and Decomposition and Space-time Coordination Modeling for Interconnected Power Systems. Automation of Electric Power Systems, 2009, 33(15):56-61.

[5] X.L. Li, M.H. Huang, L.X. Wei, et al. Multi-Regional Power Network Model Online Splicing and Merging. Automation of Electric Power Systems, 2009, 33(22):49-52
[6] Y.Z. Xin, W.M. Mi, G.D. Jiang, et al. Scheme of Application Model Information Sharing Between Control Centers Based on CIM/E. Automation of Electric Power Systems, 2013, 37(8):1-5.

[7] Y.M. Deng, B.M. Zhang, L. Tang. The Application of Topology Coloring Algorithm to Power System. Automation of Electric Power Systems, 1998, 22(10):7-9.

[8] J.K. Lin, L. Qin, P.P. Luo. A New Method for the Power System Topology Analysis Based on Graphic Modeling. Automation of Electric Power Systems, 2005, 29(22):54-59. 